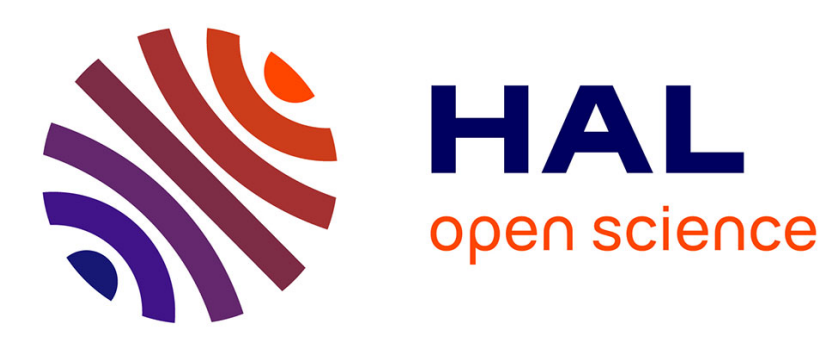

\title{
Stereological Estimation of Orientation Distribution of Generalized Cylinders from a Unique 2D Slice
} Jean-Pierre da Costa, Stefan Oprean, Pierre Baylou, Christian Germain

\section{To cite this version:}

Jean-Pierre da Costa, Stefan Oprean, Pierre Baylou, Christian Germain. Stereological Estimation of Orientation Distribution of Generalized Cylinders from a Unique 2D Slice. Microscopy and Microanalysis, 2013, 19, pp.1678-1687. 10.1017/S1431927613013548 . hal-00904011

\section{HAL Id: hal-00904011 \\ https://hal.science/hal-00904011}

Submitted on 13 Nov 2013

HAL is a multi-disciplinary open access archive for the deposit and dissemination of scientific research documents, whether they are published or not. The documents may come from teaching and research institutions in France or abroad, or from public or private research centers.
L'archive ouverte pluridisciplinaire HAL, est destinée au dépôt et à la diffusion de documents scientifiques de niveau recherche, publiés ou non, émanant des établissements d'enseignement et de recherche français ou étrangers, des laboratoires publics ou privés. 


\title{
Stereological estimation of the orientation distribution of generalized cylinders from a unique 2D slice
}

\author{
Jean-Pierre Da Costa ${ }^{\mathrm{a}, \mathrm{b}, *}$, Stefan Oprean ${ }^{\mathrm{b}}$, Pierre Baylou $^{\mathrm{a}, \mathrm{b}}$, Christian \\ Germain $^{\mathrm{a}, \mathrm{b}}$ \\ ${ }^{a}$ Univ. Bordeaux, IMS, UMR 5218, F-33400 Talence, France \\ ${ }^{b}$ CNRS, IMS, UMR 5218, F-33400 Talence, France
}

\section{Abstract}

Though 3D imaging gives a deep insight of the inner structure of complex materials, the stereological analysis of $2 \mathrm{D}$ snapshots of material sections is still necessary for large scale industrial applications for obvious reasons related to time and cost constraints. In this paper, we propose an original framework which aims at estimating the orientation distribution of generalized cylindrical structures from a single 2D section. Contrary to existing approaches, the knowledge of the cylinder cross section shape is not necessary. The only requirement is to know the area distribution of the cross sections. The approach relies on the minimization of a least squares criterion under linear equality and inequality constraints that can be solved with standard

\footnotetext{
*Corresponding author: J.P. Da Costa, IMS, 351 Cours de la Libération, 33405 Talence cedex, France.

Email address: jean-pierre.dacosta@ims-bordeaux.fr (Jean-Pierre Da Costa)
} 
optimization solvers. It is evaluated on synthetic data, including simulated images, and is applied to experimental microscopy images of fibrous composite structures. The results show the relevance and the capabilities of the approach though some limitations have been identified regarding the sensitivity to deviations from the assumed model.

Keywords: stereology, cylinder, orientation, fibrous structures, material

science

\section{Introduction}

In material science and engineering, exploring the three dimensional (3D) structure of materials is essential to understand and predict their physical properties and behaviour (e.g. Couégnat et al., 2010). In the case of composite structures, parameters of interest are for instance the volume ratio, the shapes, the sizes and the spatial distribution of the objects composing the material. In particular, the mechanical properties of woven fibrous composites closely depend on the actual 3D layout of its fibers. Even if fiber layout is meant to be consistent with the nominal manufacturing process, it may show some discrepancies in practice and has to be controlled by artificial vision. Material structure can be observed and analysed quantitatively. Resulting data can be used 
either directly or indirectly for simulations of material life (Kim et al., 1997; Davidson et al., 1997; Coindreau and Vignoles, 2005; Couégnat et al., 2010). Obviously, 3D imaging techniques can give direct insights of the material volume. However, depending on the material and on the application of interest, such imaging techniques may be too expensive and not technically appropriate. For instance, when imaging composite structures at microscopic scale, the use of tomography requires a compromise between resolution and sample size. Studies carried out on very high resolution X-ray Computarized Micro Tomography (CMT) scans $(\approx 1-2 \mu m$ per pixel) allow a fine analysis of fiber size, orientation and density at the intra-yarn scale (e.g. Coindreau and Vignoles, 2005; Martín-Herrero and Germain, 2007; Chapoullié et al., 2013). At such scales individual fibers can indeed be segmented and described but the weave itself (i.e. the mutual arrangement of yarns) cannot be investigated since only small samples of a few millimeters can be digitized. Instead, when using CMT scans at lower resolution $(\approx 10 \mu m$ per pixel) weave geometry can be analyzed but individual fibers can not be isolated and described (e.g. Badel et al., 2009; Hivet et al., 2010; Bale et al., 2012). 
An alternate way to obtain 3D images of a material is confocal microscopy (Eberhardt and Clarke, 2001; Lee et al., 2001). It consists in reconstructing 3D views from multiple optical sections of the sample. However, the use of confocal microscopy requires expensive equipment, implies specific sample preparation and applies only to semi-transparent materials. Besides, as explained by Clarke et al. (2012), confocal laser scan microscopy is of little use for characterising carbon fibres in composites unless the particular samples of interest have low packing fractions of carbon fibres.

The technique of Coherent Diffraction Imaging has also taken off in the past decade, which allows $3 \mathrm{D}$ imaging of a wide range of material and biological samples (Miao et al., 1999, 2012). Though powerful, this technique is intended for nanoscale structures and is inappropriate in industrial contexts where large material samples have to be analyzed quantitatively.

Another simple approach that does not need 3D imaging is the dissector (Sterio, 1984; Davidson et al., 1997; Lee et al., 2002). This is a stereological technique based on the observation of thin, parallel, contiguous slices of the material. However, though it generally brings worthwhile results, obtain- 
ing perfectly parallel slices of an acceptable thickness is quite laborious in practice.

Whatever the technique, investigating material structures in $3 \mathrm{D}$ reveals complex and not really appropriate in industrial contexts where imaging devices are needed to quantify and control samples both at large scale and high resolution. In such cases, 2D devices such as SEM or optical imaging are much more affordable and easy to use. Provided that microscopes are equipped with motorized stages, very large samples can be observed and analyzed with reasonable time and cost constraints. Although acquired data are two-dimensional, valuable information about 3D materials from 2D images. Stereological techniques allow to extract 3D quantitative measurements from plane sections, especially in the case of 3D objects with a simple geometry such as spheres, ellipsods or cylinders (Russ and Dehoff, 2000; Oakeshott and Edwards, 1992). When dealing with anisotropic structures, even 3D orientation can be deduced from 2D. For instance, in the case of fibre composites (Blanc et al., 2006) or cubic metallic structures (Germain et al., 2005; Blanc et al., 2010), hypotheses about the shape and spatial distribution of objects allow to derive formulas relating 3D geometry to the shape and spatial layout of $2 \mathrm{D}$ sections. 
In this paper, we deal with fibrous structures where fibres can be modelled by generalized cylinders with arbitrary shape and area. We are interested in the 3D orientations of these cylinders from 2D. More precisely, we focus on their cutting angle i.e. the angle formed between their 3D axis and the normal to the cutting plane. The relation between the cutting angle and the 2D section shape is well known when the cylinder base (i.e cross-section) is circular (Blanc et al., 2006). Indeed, in the latter case, the cylinder oblique section is an ellipse. The cosine of the cutting angle is the ratio of the minor and major axes of the ellipse. It is even possible to find the orientation of the cylinder axis up to an orientation ambiguity, which can be overcome in some cases (Mlekusch, 1999; Blanc et al., 2006).

Things get worse when the cylinder base is not circular since, in the general case, it is difficult to predict the shape of the cylinder section in $2 \mathrm{D}$ when it is cut at an arbitrary orientation. As a consequence, it is almost impossible to deduce individual cylinder axis orientation except in some simple specific cases (e.g. cylinders with triangular or rectangular bases with known geometry and area). In this paper, we argue that it is possible to deduce the statistical distribution of cutting angles from the distribution of the areas observed on an oblique section. This can be done, whatever the shapes of 
the cylinder bases, if the statistical distribution of base areas is known. Even if this approach does not retrieve individual cutting angles, it provides their statistical distribution thus allowing to quantify 3D anisotropy from 2D.

In section 2, we describe the theoretical background of the proposed approach, from the definitions and hypotheses to the implemented algorithms. In section 3 , the approach is validated through a series of experiments performed on theoretical distributions and synthetic data. In section 4, we apply the procedure to the estimation of fiber orientations in fibrous composites observed in 2D. Finally, we give a few conclusions and prospects.

\section{Theoretical background}

\subsection{A few definitions}

The word cylinder has a number of related meanings (e.g. Kern and Bland, 1948; Ballard and Brown, 1982; Harris and Stocker, 1998; Weisstein, 2013). One of the most general is the following: a cylinder or cylindrical surface is a ruled surface formed by a straight line or ruling moving continuously in 3D space without changing its direction. When the moving line returns to its starting point, the cylindrical surface is said to be closed. In this case, the curve of intersection between the cylin- 
drical surface and any plane perpendicular to the rulings is a closed curve, called cross section or base. A cylinder whose cross section is a circle or an ellipse is called a circular or an elliptic cylinder respectively.

We will call generalized cylinder a closed cylindrical surface with arbitrary cross section. We will call oblique section, the curve of intersection between the cylinder and an arbitrary cutting plane. If the cutting angle, i.e. the angle between the normal to the plane and the rulings, is different from $\pi / 2$ then the oblique section is closed and its area is finite.

Let $C$ be a random generalized cylinder, cut by an arbitrary plane. Let $X$ and $Y$ be two real valued positive random variables that represent the areas of the cross and the oblique sections of $C$ respectively. Let $\Theta \in\left[0, \frac{\pi}{2}\right]$ represent the cutting angle, as depicted in figure 1.

These three variables are linked by the following simple geometric relation:

$$
Y=\frac{X}{\cos \Theta}, \forall \Theta \in\left[0, \frac{\pi}{2}[\right.
$$

For greater simplicity, let $Z=\cos \Theta$ be the random variable describing the cosine of the cutting angle. Then :

$$
\left.\left.Y=\frac{X}{Z}, \forall Z \in\right] 0,1\right]
$$

Let $F_{X}(x)=P(X<x)$ be the cumulative distribution function of $X$ 

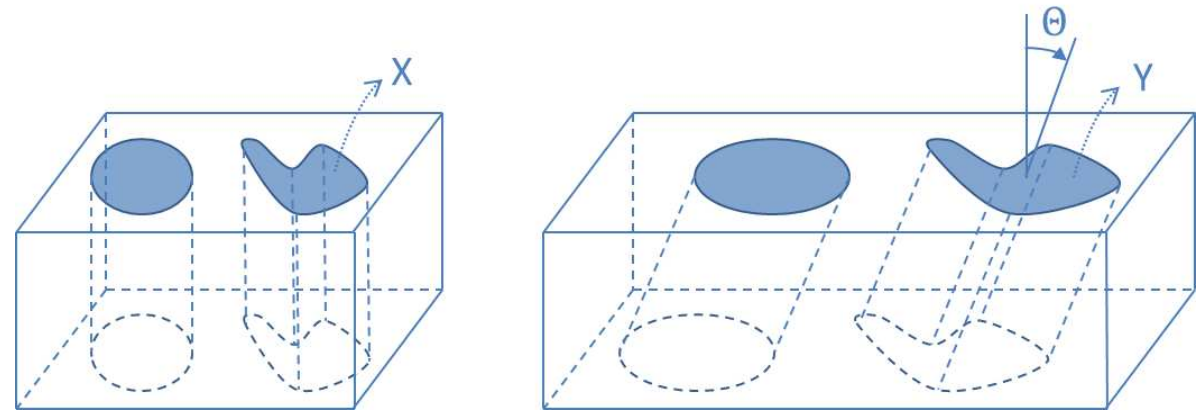

Figure 1: Cross (left) and oblique (right) sections of a circular and a generalized cylinder. $X$ denotes the area of the cross section. $Y$ is the area of the oblique section. $\Theta$ is the cutting angle i.e. the angle between the cylinder direction and the normal to the cutting plane

and $p_{X}(x)=\frac{d}{d x} F_{X}(x)$ its probability density function. Likewise, let $p_{Y}$, $p_{\Theta}, p_{Z}, F_{Y}, F_{\Theta}$ and $F_{Z}$ be the probability density functions and cumulative distribution functions of $Y, \Theta$ and $Z$.

$p_{\Theta}(\theta)$ is defined for all $\theta \in\left[0, \frac{\pi}{2}\left[\right.\right.$ while $p_{Z}(z)$ is defined for all $\left.\left.z \in\right] 0,1\right]$.

\subsection{Relating probability density functions through a differential equation}

In this paragraph, we will relate the density of the oblique section to the density of the cross section. Let us start from the expression of the cumulative:

$$
F_{Y}(y)=P(Y<y)=P\left(\frac{X}{Z}<y\right)=P(X<y Z)
$$


Conditioning on $Z$, it follows:

$$
F_{Y}(y)=\int_{0}^{1} P(X<y z \mid Z=z) p_{Z}(z) d z
$$

As one can reasonably assume that the cosine of the cutting angle and the cross section area are independent, i.e. $X$ and $Z$ are independent, the previous expression becomes:

$$
F_{Y}(y)=\int_{0}^{1} P(X<y z) p_{Z}(z) d z=\int_{0}^{1} F_{X}(y z) p_{Z}(z) d z
$$

Deriving $F_{Y}$, we get:

$$
\begin{aligned}
p_{Y}(y) & =\frac{d}{d y} F_{Y}(y)=\int_{0}^{1} \frac{\partial}{\partial y} F_{X}(y z) p_{Z}(z) d z \\
& =\int_{0}^{1} z F_{X}^{\prime}(y z) p_{Z}(z) d z \\
& =\int_{0}^{1} z p_{X}(y z) p_{Z}(z) d z
\end{aligned}
$$

Equation 6 shows that it is possible to relate the three density functions. Suppose that the densities of $\mathrm{X}$ and $\mathrm{Y}$ are known, then looking for the density of the (cosines of the) cutting angles is equivalent to finding a solution $f$ to the following differential equation:

$$
p_{Y}(y)=\int_{0}^{1} z p_{X}(y z) f(z) d z, \quad \forall y \in \mathbb{R}^{+}
$$

with the double constraint:

$$
f(z) \geq 0 ; \forall z \in[0,1] \quad \text { and } \quad \int_{0}^{1} f(z) d z=1 .
$$




\subsection{Solving the differential equation}

\subsubsection{A constrained optimization problem}

The resolution of the differential equation 7 could be addressed as a constrained optimization problem over a function space. The solution could then be looked for as a real positive function $f:[0,1] \rightarrow \mathbb{R}^{+}$with unit integral, i.e. a probability density function.

A possible optimization criterion $\Phi(f)$ to be minimized should measure how much $\int_{0}^{1} z p_{X}(y z) f(z) d z$ is in adequacy with $p_{Y}(y)$, supposedly known or estimated, whatever $y \in \mathbb{R}^{+}$. For instance, $\Phi(f)$ could be a measure of similarity between probability density functions (e.g. Kullback Leibler divergence, $L^{2}$-norm, etc.) The optimization constraints are given by equation 8.

However, it appears that such a resolution is difficult to address as it is hardly possible to choose a function space in which to perform the optimization without any a priori about the distributions of cutting angles $p_{\Theta}$ and cosines $p_{Z}$. Besides, in practice, the distribution of areas $p_{Y}$ with oblique sections is not known analytically. However it can be approximated by a discrete histogram. 


\subsubsection{Discretization of the density functions}

Let $h_{Y}=\left(h_{Y, 1}, \ldots, h_{Y, M}\right)^{t}$ be the discrete histogram of variable $Y$, i.e. the discretization of $p_{Y}$ into $M$ intervals:

$$
h_{Y, k}=\int_{y_{k}}^{y_{k+1}} p_{Y}(y) d y, \quad \forall k \in\{1, \ldots, M\}
$$

where $y_{k} \leq y_{k+1}, y_{1}=0$ and $y_{M+1}=\infty$. The operator ${ }^{t}$ denotes the matrix transpose.

$p_{Z}$ can also be discretized into $N$ classes, yielding vector $h_{Z}=\left(h_{Z, 1}, \ldots, h_{Z, N}\right)^{t}$ :

$$
h_{Z, l}=\int_{z_{l}}^{z_{l+1}} p_{Z}(z) d z, \quad \forall l \in\{1, \ldots, N\}
$$

with $z_{l} \leq z_{l+1}, z_{1}=0$ and $z_{N+1}=1$.

It comes:

$$
\begin{aligned}
h_{Y, k} & =\int_{y_{k}}^{y_{k+1}} \int_{0}^{1} z p_{X}(y z) p_{Z}(z) d z d y \\
& =\int_{y_{k}}^{y_{k+1}}\left[\sum_{l=1}^{N} \int_{z_{l}}^{z_{l+1}} z p_{X}(y z) p_{Z}(z) d z\right] d y .
\end{aligned}
$$

If the interval $\left[z_{l}, z_{l+1}\left[\right.\right.$ is small enough, $p_{Z}(z)$ can be considered to be constant over the interval, that is:

$$
p_{Z}(z) \approx \frac{h_{Z, l}}{z_{l+1}-z_{l}}, \quad \forall z \in\left[z_{l}, z_{l+1}[.\right.
$$


Then,

$$
\begin{aligned}
h_{Y, k} & \approx \int_{y_{k}}^{y_{k+1}}\left[\sum_{l=1}^{N} \int_{z_{l}}^{z_{l+1}} z p_{X}(y z) \frac{h_{Z, l}}{z_{l+1}-z_{l}} d z\right] d y \\
& =\int_{y_{k}}^{y_{k+1}}\left[\sum_{l=1}^{N} \frac{h_{Z, l}}{z_{l+1}-z_{l}} \int_{z_{l}}^{z_{l+1}} z p_{X}(y z) d z\right] d y \\
& =\sum_{l=1}^{N} h_{Z, l}\left(\frac{1}{z_{l+1}-z_{l}} \int_{y_{k}}^{y_{k+1}} \int_{z_{l}}^{z_{l+1}} z p_{X}(y z) d z d y\right)
\end{aligned}
$$

It appears that, under the assumption 12, the discrete values $h_{Y, k}$ are linked to the discrete values $h_{Z, l}$ through a linear equation:

$$
h_{Y, k} \approx \sum_{l=1}^{N} h_{Z, l} b_{k, l}
$$

with:

$$
b_{k, l}=\frac{1}{z_{l+1}-z_{l}} \int_{y_{k}}^{y_{k+1}} \int_{z_{l}}^{z_{l+1}} z p_{X}(y z) d z d y .
$$

\subsubsection{Least squares formulation}

In practice, the discrete distribution of oblique section areas $h_{Y}$ is unknown but is estimated from a statistical sample (from an image). Let $\hat{h}_{Y}=\left(\hat{h}_{Y, 1}, \ldots, \hat{h}_{Y, M}\right)^{t}$ be its estimate.

Besides, if the distribution of orthogonal section areas $p_{X}$ is known, the coefficients $b_{k, l}$ can be calculated or, at least, numerically approximated.

Our optimization problem can thus be expressed as the search of a dis- 
tribution $\hat{h}_{Z}=\left(\hat{h}_{Z, 1}, \ldots, \hat{h}_{Z, N}\right)^{t}$ so that:

$$
\hat{h}_{Y, k} \approx \sum_{l=1}^{N} \hat{h}_{Z, l} b_{k, l} \quad \forall k \in\{1, \ldots, M\}
$$

When choosing the least squares criterion, the searched distribution $\hat{h}_{Z}$ is the solution of the constrained optimization problem:

$$
\hat{h}_{Z}=\left(\hat{h}_{Z, 1}, \ldots, \hat{h}_{Z, N}\right)^{t}=\underset{r_{1}, \ldots, r_{N}}{\arg \min } \sum_{k=1}^{M}\left(\hat{h}_{Y, K}-\sum_{l=1}^{N} r_{l} b_{k, l}\right)^{2}
$$

with $\sum_{l=1}^{N} r_{l}=1$ and $r_{l} \geq 0, \forall l$.

\subsubsection{Matrix form and algorithms}

The above least squares optimization problem can be rewritten under the matrix form:

$$
\hat{h}_{Z}=\underset{R \in[0,1]^{N}}{\arg \min }\left\|h_{Y}-B R\right\|^{2}=\underset{R \in[0,1]^{N}}{\arg \min }\left(h_{Y}-B R\right)^{t}\left(h_{Y}-B R\right)
$$

with $B=\left(\begin{array}{ccc}b_{1,1} & \ldots & b_{1, N} \\ \vdots & b_{k, l} & \vdots \\ b_{M, 1} & \ldots & b_{M, N}\end{array}\right)$ and $R=\left(\begin{array}{c}r_{1} \\ \vdots \\ r_{N}\end{array}\right)$,

under the constraints $R^{t}\left(\begin{array}{c}1 \\ \vdots \\ 1\end{array}\right)=1$ and $r_{l} \geq 0, \forall l$. 
Such a quadratic optimization problem with linear equality and inequality constraints can be solved using standard algorithmic solutions such as, for instance, the functions $l s q l i n()$ or quadprog () under Matlab ${ }^{\circledR}$ environment.

\section{Experimental validation}

\subsection{Experimental design}

The proposed approach has been validated at three levels:

- Level 1, the theoretical level, focuses on the validation of the optimization procedure itself and tries to answer the following questions: is the simplification in equation 12 relevant? Do parameters $\mathrm{M}$ and $\mathrm{N}$ have a critical influence on the cutting angle distribution estimation? In order to answer these questions regardless of image digitization effects and area estimation errors, the experimental validation is based upon numerical computation. Given $p_{\Theta}$ the distribution of the cutting angles and $p_{X}$ the distribution of the cross section areas, we compute numerically $p_{Z}, p_{Y}, B$ and the theoretical discrete distributions $h_{Z}^{\text {th }}$ and $h_{Y}^{\text {th }} . B$ and $h_{Y}^{\text {th }}$ are then fed into the optimization solver (see Eq. (18)) which brings the estimate $\hat{h}_{Z}^{\text {th }}$ that can be compared with $h_{Z}^{\text {th }}$. 
- Level 2, the sampling level, evaluates the influence of statistical sampling. Our goal is here to simulate a collection of $L$ generalized cylinders with various cross sections and cutting angles. $p_{X}$ and $p_{\Theta}$ are thus sampled $L$ times providing samples for the cross section areas $\left\{x_{1}, \cdots, x_{L}\right\}$, for the oblique section areas $\left\{y_{1}, \cdots, y_{L}\right\}$, for the cutting angles $\left\{\theta_{1}, \cdots, \theta_{L}\right\}$ and their cosines $\left\{z_{1}, \cdots, z_{L}\right\}$. These samples are split into classes yielding vectors $h_{Z}^{s m p}$ and $h_{Y}^{s m p} . h_{Y}^{s m p}$ is fed into the optimization solver together with matrix $B$. The solver produces the estimate $\hat{h}_{Z}^{\text {smp }}$ that can be compared with $h_{Z}^{\text {th }}$.

- Level 3, the image level, has to deal with image synthesis and aims at evaluating the effect of digitization. The former samples for cross section area $X$ and cutting angle $\Theta$ are used to produce synthetic 2D images that simulate the oblique section of a collection of generalized cylinders. The produced binary images are labelled into various objects the areas of which are split into classes yielding vector $h_{Z}^{i m g}$ and $h_{Y}^{i m g}$. $h_{Y}^{i m g}$ is fed into the optimization solver. Its solution $\hat{h}_{Z}^{i m g}$ is finally compared with $h_{Z}^{\text {th }}$. For simplicity and without loss of generality, we have simulated circular cylinders since 2D sections can be easily simulated by drawing ellipses in an image i.e. without generating 3D data. 
In our experiments, the distribution of the cross section area is chosen to be normal: $X \sim \mathcal{N}\left(\mu_{X}, \sigma_{X}\right)$. Though arbitrary, this hypothesis proves to be relevant in practical cases (see section 4). It is always assumed to be known.

As for the cutting angles, two kinds of distributions are considered: a wrapped normal distribution $\mathcal{W N}\left(\mu_{\theta}, \sigma_{\theta}\right)$ or a mixture of two wrapped normal distributions $\Theta \sim \alpha_{1} \mathcal{W N}\left(\mu_{\theta, 1}, \sigma_{\theta, 1}\right)+\alpha_{2} \mathcal{W} \mathcal{N}\left(\mu_{\theta, 2}, \sigma_{\theta, 2}\right)$.

The proposed optimization process provides with the estimated discrete cosine distribution $\hat{h}_{Z}$ that minimizes the least squares criterion $\left\|h_{Y}-B R\right\|^{2}$. The latter measures the adequacy between the actual oblique section area distribution $h_{Y}$ and its approximation using $\hat{h}_{Y}=B \hat{h}_{Z}$. Along with this criterion, we also plot a direct measure of the adequacy between $\hat{h}_{Z}$ and $h_{Z}$ using the error function:

$$
E\left(h_{Z}, \hat{h}_{Z}\right)=\sum_{n=1}^{N}\left|h_{Z, n}-\hat{h}_{Z, n}\right| .
$$

Various values are tested for the histogram bin numbers $M$ (from 30 to 60 ) and $N$ (from 1 to $M$ ). The intervals for the area and for the angle distributions are of equal width. Simulations were performed under Matlab ${ }^{\odot}$. 

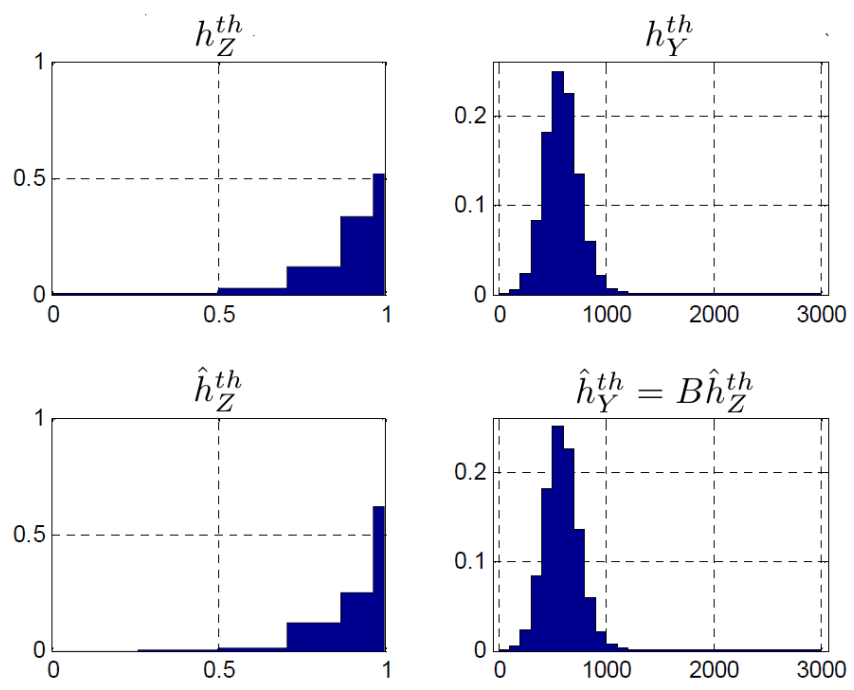

Figure 2: Comparison of input and output distributions at validation level 1. Input cutting angles $\Theta$ follow a wrapped normal distribution $\mathcal{W N}(0.2,0.3)$. Input cross sections follow a normal distribution $N(548,139)$. Other parameters are $N=6$ and $M=30$. Top left: input cosine distribution $h_{Z}^{t h}$. Bottom left: output cosine distribution $\hat{h}_{Z}^{\text {th }}$. Top right: input oblique area distribution $h_{Y}^{\text {th }}$. Bottom right: output oblique area distribution approximation $\hat{h}_{Y}^{t h}=B \hat{h}_{Z}^{t h}$.

\subsection{Results}

\subsubsection{Validation level 1: theoretical level}

Figure 2 illustrates the validation results obtained by feeding the optimization algorithm with a discrete distribution $h_{Y}^{\text {th }}$ computed numerically from a reference cross section distribution and a reference cutting angle distribution. Input cross sections follow a normal distribution $N(548,139)$. Input 


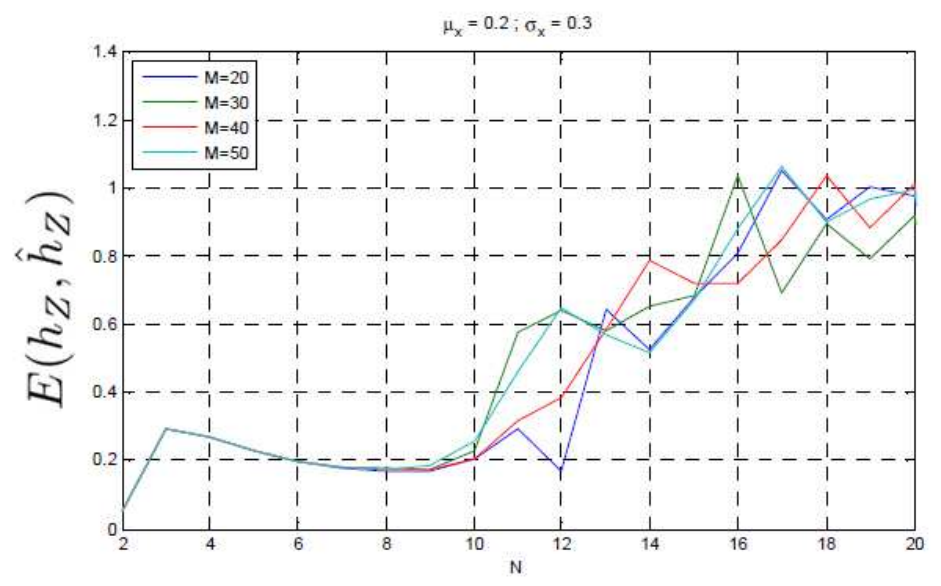

Figure 3: Plot of the error function $E\left(h_{Z}, \hat{h}_{Z}\right)$ for various bin number values $N$ and $M$. Input cutting angles $\Theta$ follow a wrapped normal distribution $\mathcal{W N}(0.2,0.3)$. Input cross sections follow a normal distribution $N(548,139)$.

cutting angles $\Theta$ follow a wrapped normal distribution $\mathcal{W N}(0.2,0.3) . \quad N=6$ and $M=30$ bins are used for angle cosines and oblique section area distributions. The optimization algorithm provides an estimated cosine distribution $\hat{h}_{Z}^{\text {th }}$ (bottom left) which is compared with the reference cosine distribution $h_{Z}^{\text {th }}$ (top left). These two distributions appear to be quite similar. Only small differences can be noticed regarding the two intervals $\Theta \in\left[0, \frac{\pi}{6}[\right.$ and $\Theta \in\left[\frac{\pi}{6}, \frac{\pi}{3}\left[(\right.\right.$ i.e. $\left.Z \in] \cos \frac{\pi}{3}, \cos \frac{\pi}{6}\right]$ and $\left.\left.\left.Z \in\right] \cos \frac{\pi}{6}, 1\right]\right) . \hat{h}_{Z}^{t h}$ is the optimal solution obtained by fitting $\hat{h}_{Y}^{\text {th }}=B \hat{h}_{Z}^{\text {th }}$ (bottom right) to $h_{Y}^{\text {th }}$ (top right). The fit between these distributions appears to be almost perfect.

In figure 3 , we plot the error $E\left(h_{Z}^{t h}, \hat{h}_{Z}^{t h}\right)$ as a function of $N$, the number 
of bins of the angle and cosine histograms, and $M$ the number of bins in the oblique section area histograms. While $M$ has not a strong influence (except for large values of $N$ ), it is shown that the quality of the estimation depends strongly on $N$. The best results are obtained with moderate values for $N$, between 6 and 9 . Though these curves relate to a specific angle distribution $\mathcal{W N}(0.2,0.3)$, similar results have been obtained with other means and standard deviations $\mu_{\theta}$ and $\sigma_{\theta}$.

Figure 4 shows another set of results obtained with a different angle distribution. A mixture of wrapped normal distributions is chosen here: $0.28 \times \mathcal{W N}(0.1,0.1)+0.72 \times \mathcal{W N}(0.4,0.3)$. Again, the fit between $h_{Y}^{\text {th }}$ and $\hat{h}_{Y}^{\text {th }}$ is very good. The solution $\hat{h}_{Z}^{\text {th }}$ is also very close to the input distribution $h_{Z}^{\text {th }}$, except from exchanges between the two intervals mentioned above.

\subsubsection{Validation level 2: statistical sampling}

The following experiment, illustrated in figure 5 , is carried out by sampling the input theoretical distributions. Cutting angles and cross section areas are sampled independently from a wrapped normal $\mathcal{W N}(0.2,0.3)$ and a normal distribution $N(548,139)$ respectively to get a total sample of 2196 angle/area pairs. Oblique section areas, obtained following equation 1, are

classified into $M$ intervals to get the discrete histogram $h_{Y}^{s m p}$. The latter is fed 
into the optimization solver which provides the estimated cosine distribution $\hat{h}_{Z}^{s m p}$. The comparison between $\hat{h}_{Z}^{s m p}$ and $h_{Z}^{s m p}$ (computed from the known angle sample) shows that the estimation is pretty good. The overall shape of the histogram is retrieved in spite of some exchanges between neighbouring intervals as mentioned previously.

This experiment was repeated a hundred times for various angle distri-
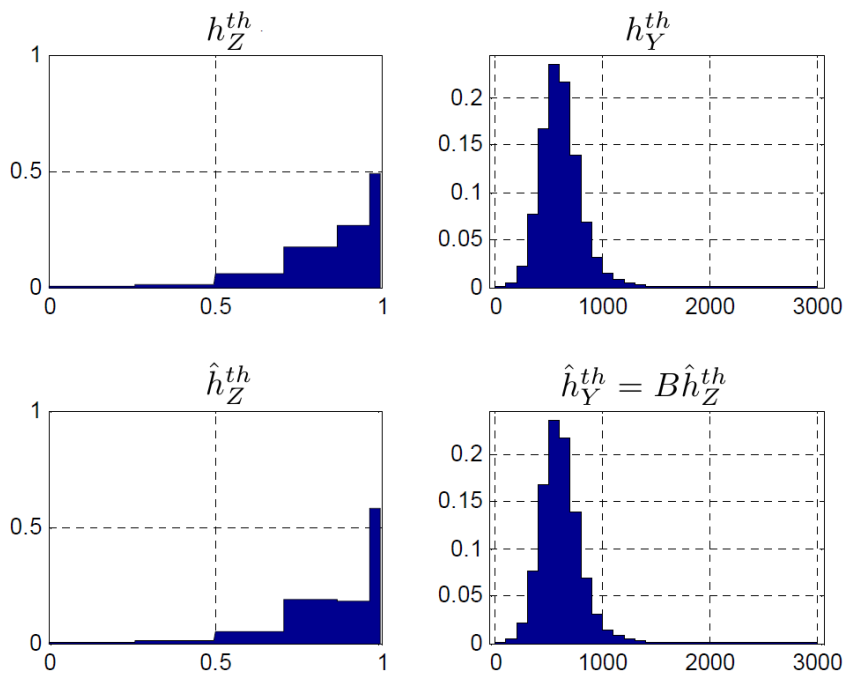

Figure 4: Comparison of input and output distributions at validation level 1. Input cutting angles $\Theta$ follow a mixture of wrapped normal distributions: $0.28 \times \mathcal{W N}(0.1,0.1)+$ $0.72 \times \mathcal{W N}(0.4,0.3)$. Input cross sections follow a normal distribution $N(548,139)$. Other parameters are $N=6$ and $M=30$. Top left: input cosine distribution $h_{Z}^{t h}$. Bottom left: output cosine distribution $\hat{h}_{Z}^{\text {th }}$. Top right: input oblique area distribution $h_{Y}^{\text {th }}$. Bottom right: output oblique area distribution approximation $\hat{h}_{Y}^{t h}=B \hat{h}_{Z}^{t h}$. 

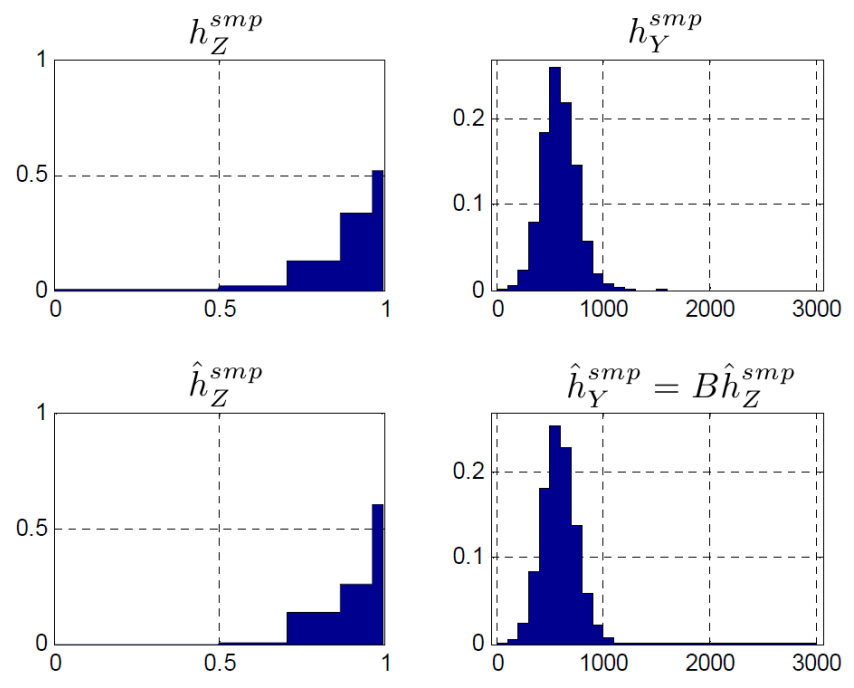

Figure 5: Comparison of input and output distributions at validation level 2. Input cutting angles $\Theta$ follow a wrapped normal distribution $\mathcal{W N}(0.2,0.3)$. Input cross sections follow a normal distribution $N(548,139)$. Other parameters are $N=6$ and $M=30$. Top left: input cosine distribution $h_{Z}^{s m p}$. Bottom left: output cosine distribution $\hat{h}_{Z}^{s m p}$. Top right: input oblique area distribution $h_{Y}^{s m p}$. Bottom right: output oblique area distribution approximation $\hat{h}_{Y}^{s m p}=B \hat{h}_{Z}^{s m p}$.

butions $W N\left(\mu_{\theta}, \sigma_{\theta}\right)$. Table 1 shows the results obtained for the least square criterion $\left\|h_{Y}^{s m p}-B \hat{h}_{Z}^{s m p}\right\|^{2}$ and the error function $E\left(h_{Z}^{t h}, \hat{h}_{Z}^{s m p}\right)$. The table values refer to the means obtained over the hundred repetitions. Comparing the various distributions, it comes that the optimization algorithm seems rather stable, whatever the angle distribution parameters. The criterion values are very low which shows an excellent fit of the oblique section histogram $h_{Y}^{s m p}$. 


\begin{tabular}{|c|c|c|c|c|c|c|c|c|c|}
\hline$\mu_{\theta}$ & 0.1 & 0.1 & 0.1 & 0.2 & 0.2 & 0.2 & 0.3 & 0.3 & 0.3 \\
$\sigma_{\theta}$ & 0.1 & 0.2 & 0.3 & 0.1 & 0.2 & 0.3 & 0.1 & 0.2 & 0.3 \\
\hline \hline$\left\|h_{Y}^{s m p}-B \hat{h}_{Z}^{s m p}\right\|^{2}$ & 0.23 & 0.26 & 0.27 & 0.27 & 0.28 & 0.26 & 0.26 & 0.24 & 0.24 \\
$\left(\times 10^{-3}\right)$ & 0.11 & 0.10 & 0.10 & 0.10 & 0.10 & 0.10 & 0.10 & 0.10 & 0.10 \\
\hline$E\left(h_{Z}^{t h}, \hat{h}_{Z}^{s m p}\right)$ & & & & & & & & & \\
\hline
\end{tabular}

Table 1: Algorithm performances for various angle distributions $W N\left(\mu_{\theta}, \sigma_{\theta}\right)$. First line: least square criterion values $\left\|h_{Y}^{s m p}-B \hat{h}_{Z}^{s m p}\right\|^{2}$. Second line: adequacy error values $E\left(h_{Z}^{t h}, \hat{h}_{Z}^{s m p}\right)$. Each value corresponds to the mean of 100 samples of size 2196.

Error value are stable too with a mean around 0.1, corresponding to small exchanges between intervals.

\subsubsection{Validation level 3: image synthesis}

The final validation stage is carried out using synthetic images that simulate the section of 3D cylindrical structures as explained above. Figure 6 shows an extract of such an image. Figure 7 reports the results obtained with distributions $X \sim \mathcal{N}(548,139), \Theta \sim \mathcal{W} \mathcal{N}(0.2,0.3)$ and samples of size 2196. Once again, the output angle distribution is very similar in shape to the input theoretical distribution. The numerical results reported in table 2 confirm both the excellent match between the observed and the estimated area distributions (i.e. $h_{y}^{i m g}$ and $\hat{h}_{y}^{i m g}=B \hat{h}_{z}^{i m g}$ ) and the good fit between the 


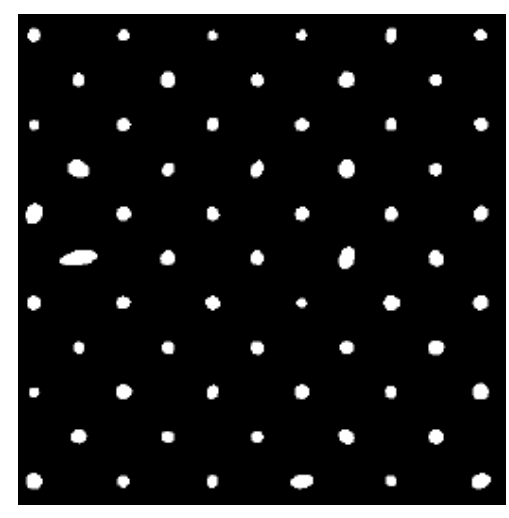

Figure 6: Extract from an example synthetic image used at validation level 3. Such an image simulates the section of a collection of cylinders whose input cross sections are distributed normally $(X \sim \mathcal{N}(548,139))$ and whose orientation cutting angle are distributed following a wrapped normal distribution $\Theta \sim \mathcal{W} \mathcal{N}(0.2,0.3)$.

input theoretical distribution and its estimation (i.e. $h_{z}^{\text {th }}$ and $\hat{h}_{z}^{i m g}$ ).

\begin{tabular}{|r|c|c|c|c|c|c|c|c|c|}
\hline$\mu_{\theta}$ & 0.1 & 0.1 & 0.1 & 0.2 & 0.2 & 0.2 & 0.3 & 0.3 & 0.3 \\
$\sigma_{\theta}$ & 0.1 & 0.2 & 0.3 & 0.1 & 0.2 & 0.3 & 0.1 & 0.2 & 0.3 \\
\hline \hline$\left\|h_{Y}^{i m g}-B \hat{h}_{Z}^{i m g}\right\|^{2}$ & 0.37 & 0.43 & 0.46 & 0.40 & 0.44 & 0.39 & 0.45 & 0.42 & 0.40 \\
$\left(\times 10^{-3}\right)$ & 0.10 & 0.10 & 0.10 & 0.10 & 0.10 & 0.10 & 0.10 & 0.10 & 0.10 \\
\hline$E\left(h_{Z}^{t h}, \hat{h}_{Z}^{i m g}\right)$ & & & & & & & & & \\
\hline
\end{tabular}

Table 2: Validation level 3: algorithm performances for various angle distributions $W N\left(\mu_{\theta}, \sigma_{\theta}\right)$. First line: least square criterion values $\left\|h_{Y}^{i m g}-B \hat{h}_{Z}^{i m g}\right\|^{2}$. Second line: error values $E\left(h_{Z}^{t h}, \hat{h}_{Z}^{i m g}\right)$. Each value corresponds to the mean of 100 samples of size 2196. 

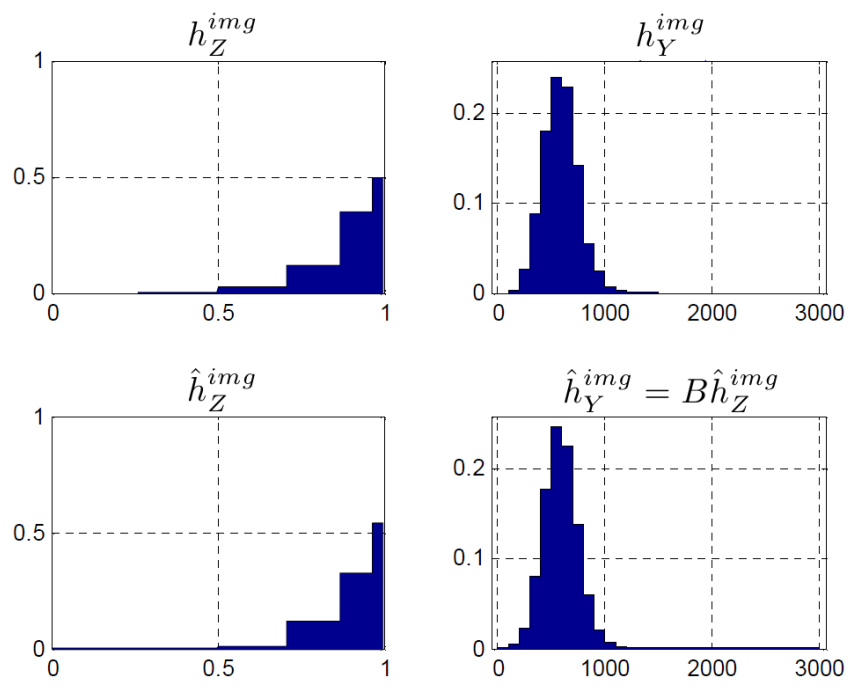

Figure 7: Validation level 3: image level. Input cutting angles $\Theta$ follow a wrapped normal distribution $\mathcal{W N}(0.2,0.3)$. Input cross sections follow a normal distribution $N(548,139)$. Other parameters are $N=6$ and $M=30$. Top left: input cosine discrete distribution $h_{Z}^{i m g}$. Bottom left: output cosine discrete distribution $\hat{h}_{Z}^{i m g}$. Top right: input oblique area discrete distribution $h_{Y}^{i m g}$. Bottom right: output oblique area discrete distribution approximation $\hat{h}_{Y}^{i m g}=B \hat{h}_{Z}^{i m g}$.

\section{Application to microscopy images of woven composite materials}

\subsection{Context}

The approach developed in this paper was applied to images of fibrous composite structures. These structures are made of threads containing around a thousand carbon fibres. The material used here is a needled laminate. It is made of woven layers composed of threads lying in the $\mathrm{X}$ and $\mathrm{Y}$ directions 


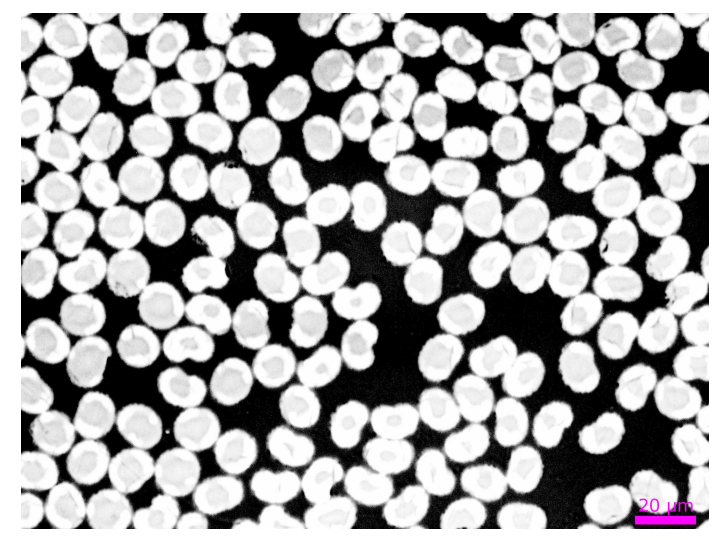

Figure 8: Micrograph of a bundle of carbon fibers with bean shapes cut orthogonally and observed by optical microscopy.

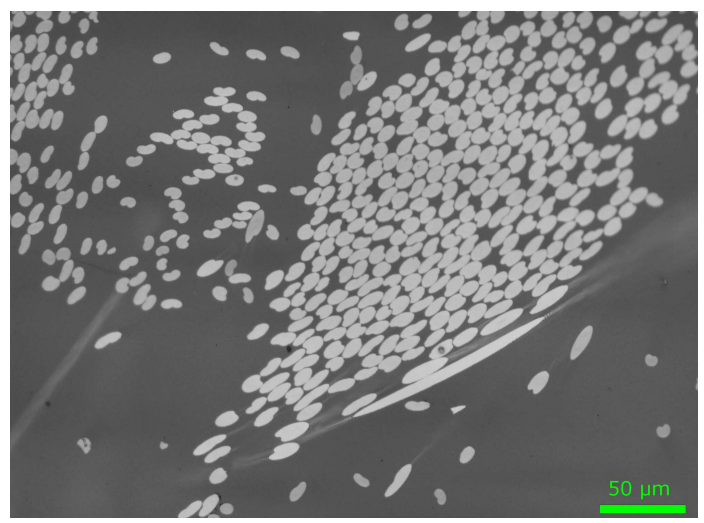

Figure 9: Micrograph of a woven composite sample showing carbon fibers with bean shapes cut at an arbitrary angle and observed by optical microscopy.

alternatively, $\mathrm{X}$ and $\mathrm{Y}$ being orthogonal. The layers are needled in a third orthogonal direction Z. Bundles in the Z direction, as they are created by the needling operation, contain fewer fibres and are less organized. However, these $\mathrm{Z}$ bundles are of great importance as regards the composite robust- 
ness. Detecting and counting $\mathrm{Z}$ fibres through microscopy imaging has thus become a routine operation for process monitoring. Material samples are imaged so that the image plane corresponds to the weaving plane. Z fibres are thus approximately orthogonal to the image plane. Z fibre sections appear as compact objects, with a more or less circular shape depending on the cutting angle but also on the initial morphology of the fibres. The simplest case is when the fibres are comparable to circular cylinders. Their 2D sections appear as ellipses, the elongation of which are closely related to the cutting angle (Blanc et al., 2006). However, when the fibres have an indeterminate shape, no simple geometric formula allows to relate the cutting angle to individual section dimensions. The latter case is illustrated in figures 8 and 9 that show two sections of such fibrous material. In figure 8, the fibres are almost perfectly orthogonal to the image plane whereas, in figure 9 , they are cut with arbitrary angles. The cross sections have clearly a non circular shape, maybe closer to a cardioid or to a bean. Oblique sections appear to have even more complex shapes. Although these shapes clearly depend on the cutting angle and on individual fibre directions in 3D, no simple geometric relation has yet been established. Better than trying to estimate individual fibre cutting angles, we then chose to estimate the global cutting 
angle distribution by applying the method presented in previous sections.

\subsection{Results and discussion}

As a prerequisite of the method is to know the distribution of fibre cross section areas, images of orthogonal views of fibre bundles were needed. As fibres rarely appear as straight parallel bundles in materials, specific samples were prepared where isolated threads were stretched and flooded with an epoxy resin by vacuum infusion. Then, these samples were cut and polished so that fibres appear as perpendicularly as possible to the sample surface. Images similar to the one in figure 8 were acquired by optical microscopy at a resolution of $0.052 \mu \mathrm{m}$ per pixel. They were finally segmented and analysed to get an estimate of the cross section area distribution.
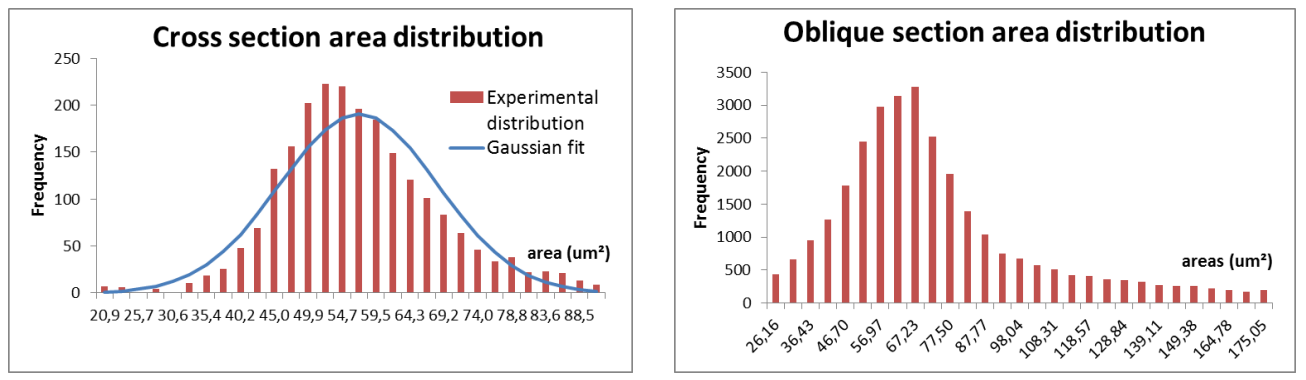

Figure 10: Area distributions. Left: cross section area distribution obtained by imaging an isolated bundle of 2,229 fibres orthogonally, at a resolution of $0.052 \mu \mathrm{m} /$ pixel. Right: cross section area distribution obtained by imaging 30, 012 fibres at a resolution of $0.26 \mu \mathrm{m} / \mathrm{pixel}$. 
Figure 10 shows, on the left, the experimental distribution and its Gaussian fit. The estimated mean and standard deviation were $57.1 \mu m^{2}$ and $11.3 \mu \mathrm{m}^{2}$ respectively. The normality of the cross section areas appeared to be a relevant assumption and that is why we made use of this assumption as we did in the experimental evaluation of the previous section. Further experiments, not showed here, revealed that the direct use of the experimental distribution instead of its Gaussian fit in the algorithms, modified only slightly the final results.

The reader may also notice that the experimental distribution is not exactly symmetric, which entails a slight deviation of the Gaussian fit to the right and a little overestimation of the distribution mode. This is due to the presence of some fibres that are not perfectly orthogonal to the image plane.

Besides the preliminary study of cross sections, a sample of a woven carbon composite was also prepared by resin vacuum infusion and analysed by microscopy. A collection of images similar to the one in figure 9 was acquired at a resolution of $0.26 \mu \mathrm{m}$ per pixel. A total of 30,012 fibres, cut at arbitrary angles, were segmented and analysed. Figure 10 shows, on the right, the observed oblique section area distribution. It can be seen that the distribution mode has now clearly 
moved to the right and that the distribution is now heavy tailed due to the presence of a high number of fibres cut with large angles.
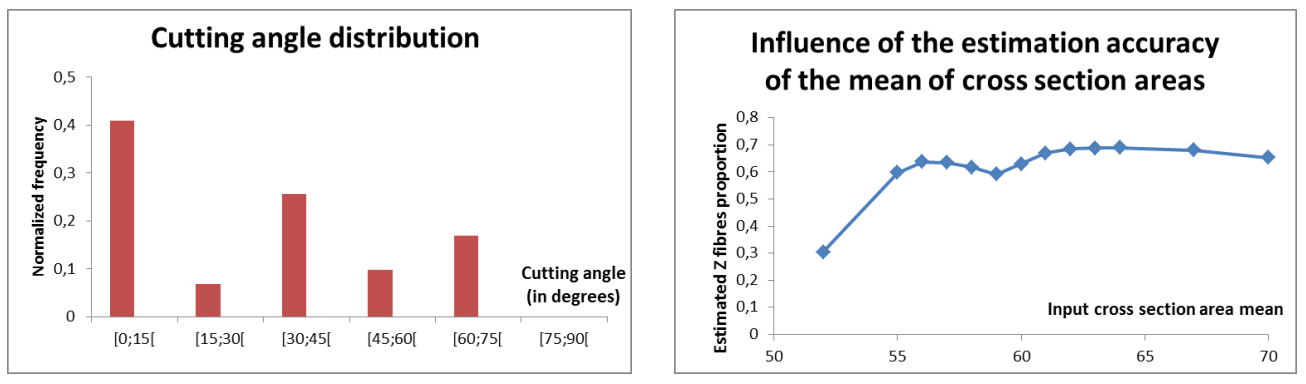

Figure 11: Angle distributions. Left: cutting angle distribution obtained from the cross section distribution and the oblique section distributions of figure 10. Right: plot of the $\mathrm{Z}$ fibres proportion as a function of the cross section area mean given as an input to the inversion algorithm.

Figure 11 (left) reports the result of the inversion algorithm proposed in this article i.e. the distribution of the angular deviates of fibres from the normal of the image plane. $\mathrm{Z}$ fibres are defined by material specialists as fibres that are less than a given angle $\theta_{\max }$ apart from the normal to the weaving plane. As the weaving plane corresponds approximately to the image plane, obtaining the $\mathrm{Z}$ fibres count is straightforward: this is done by summing the histogram bins related to angles smaller than $\theta_{\max }$ degrees. This result is thus a proof of concept that the orientation distribution of fibers considered as cylinders of 
arbitrary shapes can be retrieved from a unique 2D slice, given the knowledge of the cross section area distribution.

However, it was found that the result of the inversion algorithm was relatively dependent on the primary stage which consists in estimating the distribution of the fibre cross section areas. Indeed, it was shown that little over- or under-estimations of the area mean and standard deviations might hinder the behaviour of the algorithm. Figure 11 (right) plots the estimated Z fibres proportion as a function of the mean cross section area given as an input to the inversion algorithm. It can be seen that, in spite of the presence of a plateau around the correct mean estimate (i.e. $57-58 \mu m^{2}$ ), the algorithm shows some sensitivity to over or under estimations. Similar results were obtained concerning the standard deviation. Such sensitivity suggests that the prior estimation of cross section area distribution must be carried out carefully even if average fiber sizes are supposed to be provided by fiber suppliers. When using 2-D microscopy imaging, the estimation of the area distribution must be carried out on specific fibre bundle samples prepared and observed so that fiber sections appear as perpendicular to the image plane as possible. Actually, such orthogonality is difficult to obtain in practice and a 
special care must be taken to ensure that the fibers are perfectly straight and parallel. Though not adapted for large scale studies, 3-D imaging techniques could be used for the prior estimation of area distributions instead of 2-D imaging, thus avoiding difficulties relate to sample preparation. High resolution x-ray microtomography (Chapoullié et al., 2013) or x-ray coherent diffraction imaging (Miao et al., 2012) could be interesting alternatives.

Another potential limitation is the variability of fiber supplies. Indeed, it was observed that fiber section areas may vary from a bundle to another. As a corollary, a specific attention must be paid to how the reference sample used for area distribution estimation is representative of the actual carbon weave. The required number of fiber bundles will have to be investigated to guarantee the relevance of the distribution estimation.

\section{Conclusion}

In this paper, we have presented and evaluated a novel method for the estimation of the orientation distribution of cylindrical structures from a single section image. Contrary to existing approaches, the method is not tied 
to the shape of cylinder bases and can be applied with structures of arbitrary shapes. The only prerequisite is the knowledge of the cross section area distribution. It hinges on the discretization of area and angle distributions and on the minimization of a least squares criterion that can be carried out with standard quadratic optimization solvers. A thorough evaluation has been conducted on synthetic data which showed the capabilities of the approach in various cases including unimodal and bimodal angle distributions with various mean and standard deviations. Finally, the proposed algorithm was applied in an industrial context to the estimation of cutting angles from images of 3D fibrous structures. Here again, the capabilities of the approach were evaluated. Though promising, the approach proved to be sensitive to deviations from the model used in the algorithm. The first results obtained on experimental images showed that the prior estimation of the cross section area distribution must be carried out with accuracy. Future work will be devoted to a deeper study of the approach sensitivity both on synthetic and experimental data, and of the conditions under which it can be applied in an industrial context. 


\section{References}

Badel, P., Vidal-Sallé, E., Maire, E., Boisse, P., 2009. Simulation and tomography analysis of textile composite reinforcement deformation at the mesoscopic scale. International Journal of Material Forming 2, 189-192.

Bale, H., Blacklock, M., Begley, M.R., Marshall, D.B., Cox, B.N., Ritchie, R.O., 2012. Characterizing three-dimensional textile ceramic composites using synchrotron x-ray micro-computed-tomography. Journal of the American Ceramic Society 95, 392-402.

Ballard, D.H., Brown, C.M., 1982. Computer Vision. Prentice Hall, New Jersey, USA. chapter 9.1. pp. 274-275.

Blanc, R., Baylou, P., Germain, C., Da Costa, J.P., 2010. Confidence bounds for the estimation of the volume phase fraction from a single image in a nickel base superalloy. Microscopy and Microanalysis 16, 273-281.

Blanc, R., Germain, C., Da Costa, J.P., Baylou, P., Cataldi, M., 2006. Fiber orientation measurements in composite materials. Composites Part A 37, $197-206$.

Chapoullié, C., Germain, C., Da Costa, J., Vignoles, G.L., Cataldi, M., 
2013. Multiscale esxtraction of morphological features in woven cmcs, in: Proceedings of ICACC 2013, Daytona Beach USA.

Clarke, A., Eberhardt, C., Davidson, N., 2012. 3d characterisation of glass fibres in composites by confocal microscopy, in: Proceedings of ICCM12, Paris.

Coindreau, O., Vignoles, G.L., 2005. Assessment of structural and transport properties in fibrous c/c composite preforms as digitized by x-ray cmt. part i : Image acquisition and geometrical properties. Journal of Materials Research 20, 2328-2339.

Couégnat, G., Martin, E., Lamon, J., 2010. 3D Multiscale Modeling of the Mechanical Behavior of Woven Composite Materials. John Wiley \& Sons, Inc. pp. 185-194.

Davidson, N., Clarke, A., Archetypal, G., 1997. Large-area, high-resolution image analysis of composite materials. Journal of Microscopy 185, 233-242.

Eberhardt, C., Clarke, A., 2001. Fiber-orientation measurements in shortglass-fibre composites. part i. automated, high-angular-resolution measurement by confocal microscopy. Composites Science and Technology 61, $1389-1400$. 
Germain, C., Blanc, R., Donias, M., Lavialle, O., Da Costa, J.P., Baylou, P., 2005. Estimating the section elevation angle of cubes on a cubic mesh. application to nickel microstructure size estimation. Image Analysis \& Stereology 24, 127-134.

Harris, J.W., Stocker, H., 1998. Handbook of Mathematics and Computational Science. Springer-Verlag, New York. chapter 4.6. pp. 102-104.

Hivet, G., Wendling, A., Vidal-Salle, E., Laine, B., Boisse, P., 2010. Modeling strategies for fabrics unit cell geometryapplication to permeability simulations. International Journal of Material Forming 3, 727-730.

Kern, W.F., Bland, J.R., 1948. Solid Mensuration with Proofs. Wiley, New York. chapter 16-17. pp. 36-42.

Kim, J., Liaw, P.K., Hsu, D.K., McGuire, D.J., 1997. Nondestructive evaluation of nicalon/sic composites by ultrasonics and x-ray computed tomography. Ceram. Eng. Sci. Proc. 18, 287-296.

Lee, K.S., Lee, S.W., Youn, J., Kang, T., Chung, K., 2001. Confocal microscopy measurement of the fiber orientation in short fiber reinforced plastics. Fibers and Polymers 2, 41-50. 
Lee, Y.H., Lee, S.W., Youn, J., Chung, K., Kang, T., 2002. Characterization of fiber orientation in short fiber reinforced composites with an image processing technique. Materials Research Innovations 6, 65-72.

Martín-Herrero, J., Germain, C., 2007. Microstructure reconstruction of fibrous c/c composites from x-ray microtomography. Carbon 45, $1242-$ 1253.

Miao, J., Charalambous, P., Kirz, J., Sayre, D., 1999. Extending the methodology of x-ray crystallography to allow imaging of micrometre-sized noncrystalline specimens. Nature, $342-344$.

Miao, J., Sandberg, R., Song, C., 2012. Coherent x-ray diffraction imaging. Selected Topics in Quantum Electronics, IEEE Journal of 18, 399-410.

Mlekusch, B., 1999. Fiber orientation in short-fiber-reinforced thermoplastics. ii. quantitative measurements by image analysis. Composites Science and Technology 59, 547-560.

Oakeshott, R.B.S., Edwards, S.F., 1992. On the stereology of ellipsoids and cylinders. Physica A: Statistical Mechanics and its Applications 189, 208233. 
Russ, J., Dehoff, R., 2000. Practical Stereology. New York: Plenum Press.

Sterio, D., 1984. The unbiased estimation of number and sizes of arbitrary particles using the dissector. Journal of Microscopy 134, 127-136.

Weisstein, E.W., 2013. Cylinder, from mathworld-a wolfram web resource. url: http://mathworld.wolfram.com/Cylinder.html. 